\title{
Editorial
}

\section{Nanofluidics and Nanofluids}

\author{
Jianzhong Lin $\mathbb{D},{ }^{1}$ Mingzhou Yu, ${ }^{2}$ Martin Seipenbusch, ${ }^{3}$ Xiaoke Ku $\mathbb{D},{ }^{1}$ and Yu Feng $\mathbb{D}^{4}$ \\ ${ }^{1}$ Department of Engineering Mechanics, Zhejiang University, Hangzhou, China \\ ${ }^{2}$ College of Mechanical and Electrical Engineering, China Jiliang University, Hangzhou, China \\ ${ }^{3}$ Institute of Chemical Process Engineering, University of Stuttgart, Stuttgart, Germany \\ ${ }^{4}$ School of Chemical Engineering, Oklahoma State University, Stillwater, OK, USA \\ Correspondence should be addressed to Jianzhong Lin; mecjzlin@zju.edu.cn
}

Received 5 November 2018; Accepted 5 November 2018; Published 2 May 2019

Copyright (c) 2019 Jianzhong Lin et al. This is an open access article distributed under the Creative Commons Attribution License, which permits unrestricted use, distribution, and reproduction in any medium, provided the original work is properly cited.

\section{Introduction}

Nanofluidics is the research of the behavior, manipulation, and control of fluids which are confined to nanometer-sized structures, while nanofluids are a class of fluids which contain nanoparticles. Both nanofluidics and nanofluids can exhibit novel physical behaviors not observed in larger structures (e.g., increased viscosity, enhanced thermal conductivity, and special rheological and acoustical properties), which make them potentially useful in many applications. Recently, the advancement in nanotechnology has triggered a greater motivation to investigate nanofluidics and nanofluids in detail.

\section{Overview of the Works Published in This Special Issue}

The papers published in this special issue cover a wide range of research topics from fundamental physical concepts to applied technologies in the field of nanofluidics and nanofluids. Both experimental and modeling studies are included, and they might be broadly categorized into three groups.

The focus of the first group which consists of 4 papers is on aerosol systems. Particle size distribution (PSD) is one of the most important properties of aerosol particles. In the paper entitled "The Asymptotic Behavior of Particle Size Distribution Undergoing Brownian Coagulation Based on the Spline-Based Method and TEMOM Model" by He and Xie, the PSD was reconstructed using finite moments based on a converted spline-based method, and the evolution of PSD undergoing Brownian coagulation and its asymptotic behavior were also investigated. Liu et al. mathematically investigated the interaction of nanoparticle dynamics between modes by establishing two joint population balance equations (PBEs) in the paper entitled "Study on the Interaction between Modes of a Nanoparticle-Laden Aerosol System." In another paper entitled "Modeling of Scattering Cross Section for Mineral Aerosol with a Gaussian Beam," Zheng and Tang studied the scattering cross section of nonspherical mineral particles within the Gaussian beam based on the Generalized Lorenz-Mie Theory (GLMT). A review covering particle flow measurement and nano- and microaerosol distribution and deposition was presented by Zhou et al. in their paper entitled "Experimental Views of Tran-Bend Particle Deposition in Turbulent Flow with Nanoscale Effect." The effect of environmental humidity, particle and surface properties, nanoparticle formation, coagulation, or evolution phenomena on particle deposition was discussed.

The second group consists of 9 papers and covers a wide range of micro/nanoscale particulate matter types (e.g., solid particles, droplets, bubbles, and flexible fibers) used in different applications. To understand the expansion characteristic differences between polymeric and core-shell microspheres, Diwu et al. experimentally and mathematically investigated the expansion behaviors of these two types of microspheres and provided insightful discussions towards the advantages of using those microspheres on oil recovery. Details can be found in the paper entitled "Experimental Study on Expansion Characteristics of Core-Shell and Polymeric Microspheres." Soot formation and evolution are constantly investigated issues in combustion processes. Jiang et al. presented the paper entitled "Stochastic Simulation of 
Soot Formation Evolution in Counterflow Diffusion Flames," in which soot formation and evolution in counterflow diffusion flames were investigated. Moreover, two detailed gas kinetic mechanisms ( $\mathrm{ABF}$ and $\mathrm{KM} 2$ ) were also compared with each other. Li et al. employed the volume-offluid (VOF) method to investigate microbubble transport patterns in an ultrasound field in the paper entitled "Shape Oscillation of a Single Microbubble in an Ultrasound Field." In another paper entitled "Molecular Dynamics Simulation of Nanoscale Channel Flows with Rough Wall Using the Virtual-Wall Model," Lin et al. applied molecular dynamics simulation to study the nanoscale gas flow characteristics in rough channels. They mainly concerned the effect of roughness element geometry on flow behaviors and found that the fluid velocity decreased with increasing roughness element height. Qian et al. proposed a new design of spraycoating nozzle and quantitatively evaluated its enhanced performance of nanoparticle-coating uniformity using both numerical and experimental methods. Specific discussions can be found in the paper entitled "A New Spray Approach to Produce Uniform Ultrafine Coatings." Using the lattice Boltzmann method (LBM) in the paper entitled "TwoDimensional Numerical Study on the Migration of Particle in a Serpentine Channel," Liu et al. numerically studied the migration of particles in a serpentine channel with parametric analyses of how Reynolds number and initial particle positions could impact particle migration behaviors. They also found that there existed a critical solid-to-fluid density ratio at which the particle traveled fastest in the channel. Lin et al. utilized the flexible fiber model and large eddy simulation (LES) to simulate the transport and deformation of a micro/nanoscale fiber in a jet flow field, in the paper entitled "Simulation of Motion of Long Flexible Fibers with Different Linear Densities in Jet Flow." Yuan et al. constructed a coupled numerical model for nanorod-based suspension flow and investigated the convective heat transfer and resistance characteristics of the nanofluid duct flow in the paper entitled "Numerical Research on Convective Heat Transfer and Resistance Characteristics of Turbulent Duct Flow Containing Nanorod-Based Nanofluids." Lin and Zhang studied the motion of cylindrical nanoparticles in a mixing layer using the pseudospectral method and discrete particle model in their paper entitled "Simulation and Visualization of Flows Laden with Cylindrical Nanoparticles in a Mixing Layer." The effect of Stokes number and particle aspect ratio on the mixing and orientation distribution of cylindrical particles was analyzed.

The third group is related to flow and transport behaviors with electrical issues. For the purpose of investigating electric driving mechanism due to an induced charge electro-osmotic flow, Zhang et al. applied the finite volume method to solve the induced charge electro-osmotic flow in the paper entitled "Design and Numerical Study of Micropump Based on Induced Electroosmotic Flow." They mainly studied vortices in the flow as well as their effect on the electric double layer, and the findings might be useful for the practical application of induced electro-osmotic flow in a micropump. In Zhang and Zhang's work entitled "Iterative Dipole Moment Method for the Dielectrophoretic Particle-
Particle Interaction in a DC Electric Field," an iterative dipole moment (IDM) method was applied to study the dielectrophoretic (DEP) forces of particle-particle interactions in a two-dimensional DC electric field. The relationship between the chain patterns and the DEP properties was found by tracking each particle movement.

\section{Conclusions}

This special issue documents some new applications and challenges in the area of nanofluidics and nanofluids and the accepted papers show a diversity of new findings and overviews of the recent research and development. However, this special issue is far from an exhaustive survey of all the current topics and trends in nanofluidics and nanofluids research. Many additional important research issues of nanofluidics and nanofluids still remain to be explored in more depth.

\section{Conflicts of Interest}

The authors declare that they have no conflicts of interest.

\section{Acknowledgments}

The editors are grateful to the participants of this special issue for their inspiring contributions and the anonymous reviewers for their helpful and constructive comments which greatly improved the contents of the papers published in this special issue.

Jianzhong Lin

Mingzhou Yu

Martin Seipenbusch

Xiaoke Ku

Yu Feng 


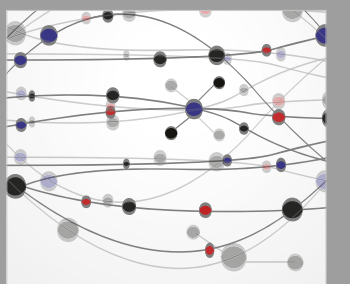

The Scientific World Journal
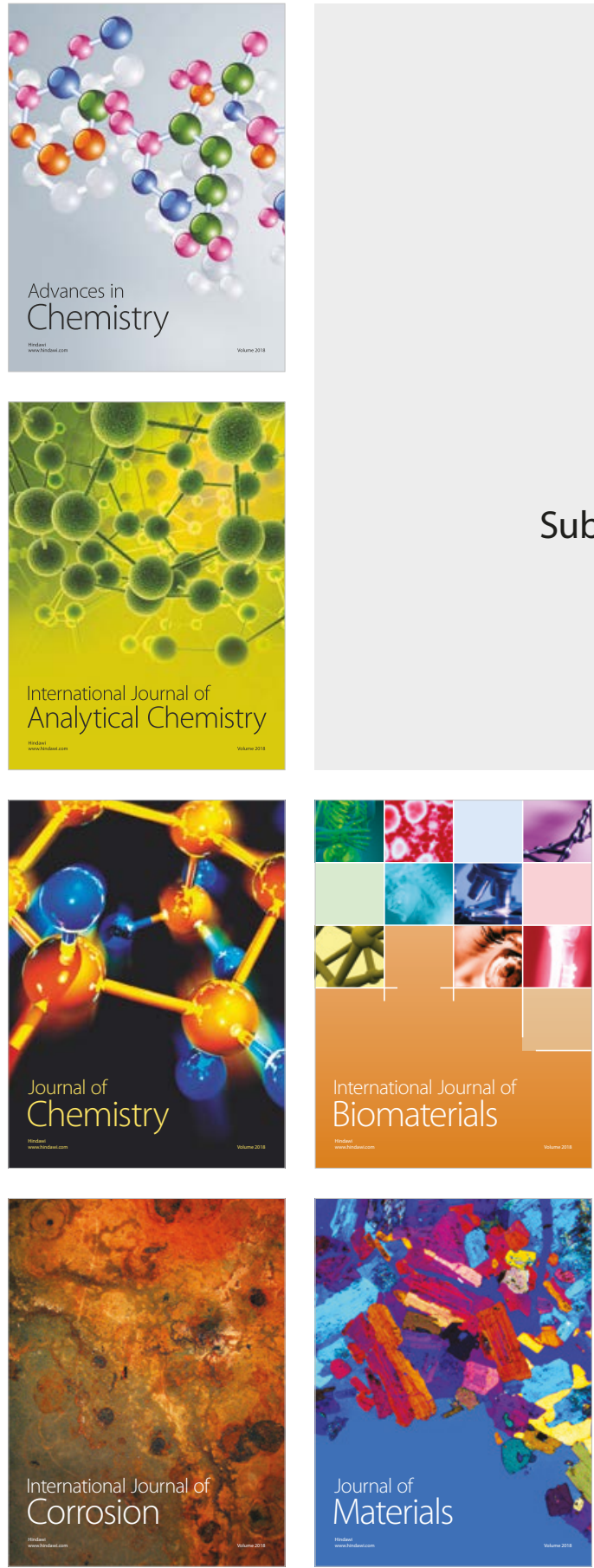

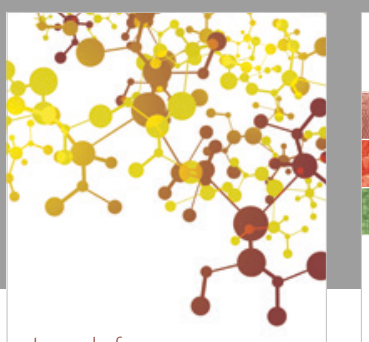

Journal of

Applied Chemistry
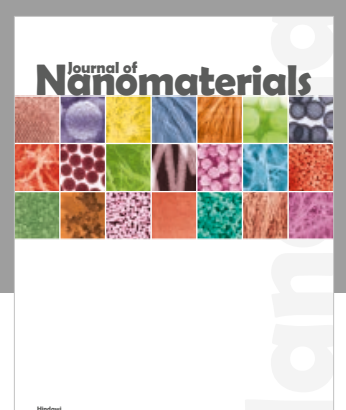

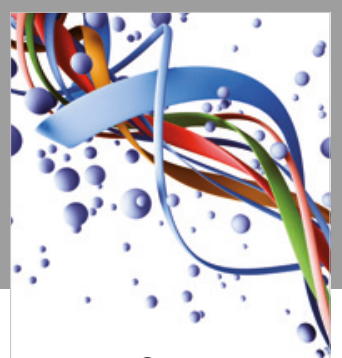

Scientifica

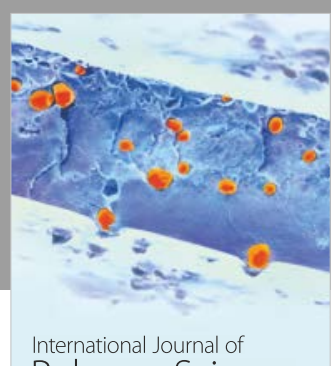

Polymer Science

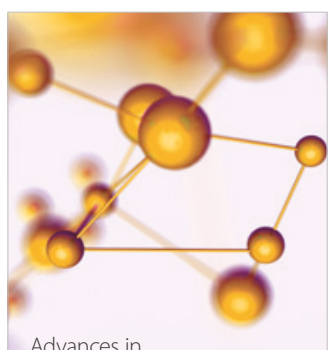

Physical Chemistry
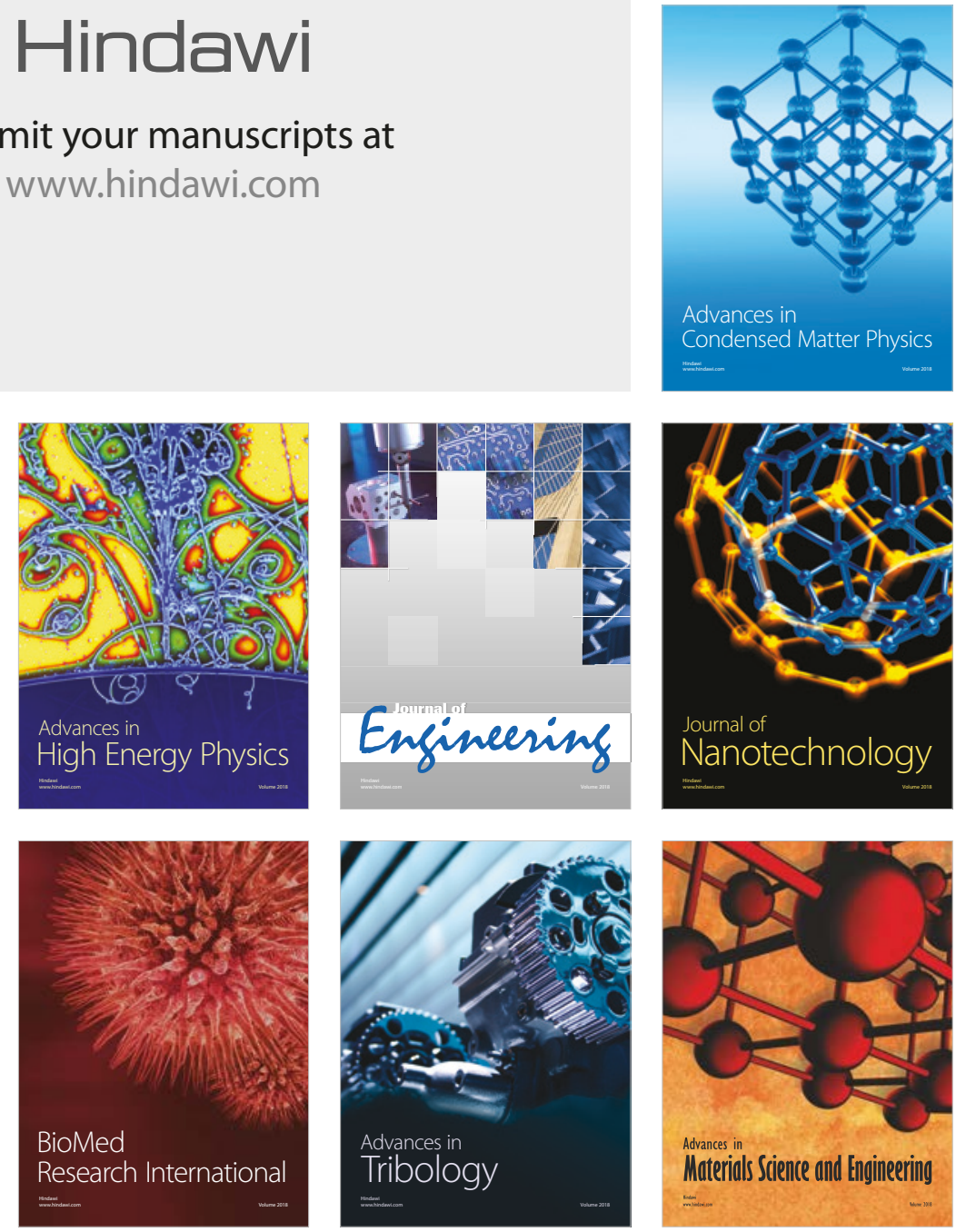